\title{
A multiplicative characterization of the power means
}

\author{
Tom Leinster*
}

\begin{abstract}
A startlingly simple characterization of the $p$-norms has recently been found by Aubrun and Nechita [2] and by Fernández-González, Palazuelos and Pérez-García [3]. We deduce a simple characterization of the power means of order $\geq 1$.
\end{abstract}

\section{Introduction}

For each real $p \neq 0$, the power mean (or generalized mean) of order $p$ assigns the quantity

$$
\left(\frac{1}{n} \sum_{i \in I} x_{i}^{p}\right)^{1 / p}
$$

to a family $\left(x_{i}\right)_{i \in I}$ of $n$ positive real numbers. More generally, uneven weights $\left(w_{i}\right)_{i \in I}$ may be attached to the arguments, giving the power mean

$$
M_{p}(w, x)=\left(\sum_{i \in I} w_{i} x_{i}^{p}\right)^{1 / p}
$$

The power mean of order 0 is defined as the limit of this expression as $p \rightarrow 0$, namely $M_{0}(w, x)=$ $\prod_{i} x_{i}^{w_{i}}$. For the same reason, one defines $M_{-\infty}(w, x)=\min _{i} x_{i}$ and $M_{\infty}(w, x)=\max _{i} x_{i}$.

The basic theory of power means is laid out in the classic text of Hardy, Littlewood and Pólya [4]. In particular, their Theorem 215, when taken in conjunction with Theorem 84, provides an axiomatic characterization of the means $M_{p}$ of order $p \in(0, \infty)$.

Here we give a different characterization, capturing the means $M_{p}$ of order $p \in[1, \infty]$. It is based on the recent characterization by Aubrun and Nechita [2] of the $p$-norms

$$
\|x\|_{p}= \begin{cases}\left(\sum_{i \in I}\left|x_{i}\right|^{p}\right)^{1 / p} & \text { if } p<\infty \\ \max _{i \in I}\left|x_{i}\right| & \text { if } p=\infty\end{cases}
$$

This formula puts a norm on $\mathbb{R}^{I}$ for each finite set $I$, and is multiplicative: if $x \in \mathbb{R}^{I}$ and $y \in \mathbb{R}^{J}$ then $\|x \otimes y\|_{p}=\|x\|_{p}\|y\|_{p}$, where $x \otimes y \in \mathbb{R}^{I} \otimes \mathbb{R}^{J} \cong \mathbb{R}^{I \times J}$. Roughly speaking, their result - which we review below - is that multiplicativity characterizes the $p$-norms uniquely. We deduce from it a multiplicative characterization of the power means.

The theorem proved by Aubrun and Nechita is very closely related to earlier results of Fernández-González, Palazuelos and Pérez-García [3], although the proofs are not at all similar. Here it will be more convenient to use Aubrun and Nechita's formulation.

Acknowledgements I thank Mark Meckes and Carlos Palazuelos for useful discussions.

${ }^{*}$ School of Mathematics and Statistics, University of Glasgow, University Gardens, Glasgow G12 8QW, UK; Tom.Leinster@glasgow.ac.uk. Supported by an EPSRC Advanced Research Fellowship. Mathematics Subject Classification (2010): 26E60 (primary), 47A30, 52A21. 


\section{Statement of the theorem}

Write $\mathbb{R}_{+}=\{x \in \mathbb{R} \mid x \geq 0\}$. For a finite set $I$, write

$$
\Delta_{I}=\left\{w \in \mathbb{R}_{+}^{I} \mid \sum_{i \in I} w_{i}=1\right\} .
$$

For each map $f: I \rightarrow J$ of finite sets, there is an induced map $\Delta_{I} \rightarrow \Delta_{J}$, denoted by $w \mapsto f w$ and defined by $(f w)_{j}=\sum_{i \in f^{-1}(j)} w_{i}$. There is also an induced map $\mathbb{R}_{+}^{J} \rightarrow \mathbb{R}_{+}^{I}$, denoted by $x \mapsto x f$ and defined by $(x f)_{i}=x_{f(i)}$. For finite sets $I$ and $J$, there are canonical maps

$$
\Delta_{I} \times \Delta_{J} \stackrel{\otimes}{\rightarrow} \Delta_{I \times J}, \quad \mathbb{R}_{+}^{I} \times \mathbb{R}_{+}^{J} \stackrel{\otimes}{\rightarrow} \mathbb{R}_{+}^{I \times J}
$$

defined by $x \otimes y=\left(x_{i} y_{j}\right)_{(i, j) \in I \times J}$ whenever $(x, y) \in \Delta_{I} \times \Delta_{J}$ or $(x, y) \in \mathbb{R}_{+}^{I} \times \mathbb{R}_{+}^{J}$.

Definition $1.1 \quad$ i. A system of means consists of a function $M: \Delta_{I} \times \mathbb{R}_{+}^{I} \rightarrow \mathbb{R}_{+}$for each finite set $I$, satisfying:

Functoriality: $M(f w, x)=M(w, x f)$ whenever $f: I \rightarrow J$ is a map of finite sets, $w \in \Delta_{I}$ and $x \in \mathbb{R}_{+}^{J}$.

Consistency: $M((1),(c))=c$ whenever $c \in \mathbb{R}_{+}$and $I$ is a one-element set, where (1) denotes the unique element of $\Delta_{I}$ and $(c)$ is the element of $\mathbb{R}_{+}^{I}$ corresponding to $c$.

Monotonicity: $M(w, x) \leq M(w, y)$ whenever $I$ is a finite set, $w \in \Delta_{I}$ and $x, y \in \mathbb{R}_{+}^{I}$ with $x_{i} \leq y_{i}$ for all $i \in I$.

ii. A system of means $M$ is convex if $M\left(w, \frac{x+y}{2}\right) \leq \max \{M(w, x), M(w, y)\}$ whenever $I$ is a finite set, $w \in \Delta_{I}$ and $x, y \in \mathbb{R}_{+}^{I}$.

iii. A system of means $M$ is multiplicative if $M(w \otimes v, x \otimes y)=M(w, x) M(v, y)$ whenever $I$ and $J$ are finite sets, $(w, x) \in \Delta_{I} \times \mathbb{R}_{+}^{I}$ and $(v, y) \in \Delta_{J} \times \mathbb{R}_{+}^{J}$.

If $M(w, \xi)$ is written as $\int \xi d w$, then functoriality becomes the classical formula for integration under a change of variables or integration against a push-forward measure:

$$
\int \xi d\left(f_{*} w\right)=\int(\xi \circ f) d w
$$

(This notation is potentially misleading, since $M(w, \xi)$ need not be linear in $\xi$.) The significance of functoriality will be explained further in the next section.

Example 1.2 For each $p \in[0, \infty]$ there is a multiplicative system of means $M_{p}$ defined by

$$
M_{p}(w, x)= \begin{cases}\prod_{i \in I} x_{i}^{w_{i}} & \text { if } p=0 \\ \left(\sum_{i \in I} w_{i} x_{i}^{p}\right)^{1 / p} & \text { if } 0<p<\infty \\ \max _{i: w_{i}>0} x_{i} & \text { if } p=\infty\end{cases}
$$

$\left(w \in \Delta_{I}, x \in \mathbb{R}_{+}^{I}\right.$ ). If $p \geq 1$ then $M_{p}$ is convex, by the triangle inequality for the $p$-norm. If $p<1$ then $M_{p}$ is not convex, as may be seen by taking $w=(1 / 2,1 / 2), x=(1,0)$ and $y=(0,1)$.

The purpose of this note is to prove:

Theorem 1.3 Every convex multiplicative system of means is equal to $M_{p}$ for some $p \in[1, \infty]$. 


\section{Proof of the theorem}

To prove their characterization theorem for $p$-norms, Aubrun and Nechita use a standard result of probability theory, Cramér's large deviation theorem, and Fernández-González, Palazuelos and Pérez-García use techniques from the theory of Banach spaces. In contrast, the deduction of our theorem from theirs is elementary and almost entirely self-contained.

We begin by recording some elementary properties of systems of means. We then take a convex multiplicative system of means, $M$, and extract a number $p \in[1, \infty]$. The proof that $M=M_{p}$ proceeds in two steps. First we make the connection between means and norms and apply the $p$-norm characterization theorem, concluding that $M$ and $M_{p}$ agree when the weighting is uniform $\left(w_{i}=w_{j}\right.$ for all $\left.i, j\right)$. Then we apply standard arguments to extend this result to uneven weightings.

\subsection{Elementary properties of systems of means}

When $I=\{1, \ldots, n\}$ for some integer $n \geq 1$, we write $\Delta_{n}$ for $\Delta_{I}$ and $\mathbb{R}_{+}^{n}$ for $\mathbb{R}_{+}^{I}$.

Lemma 2.1 Every system of means $M$ has the following properties.

i. Symmetry: for all $n \geq 1,(w, x) \in \Delta_{n} \times \mathbb{R}_{+}^{n}$, and permutations $\sigma \in S_{n}$,

$$
M(w, x)=M\left(\left(w_{\sigma(1)}, \ldots, w_{\sigma(n)}\right),\left(x_{\sigma(1)}, \ldots, x_{\sigma(n)}\right)\right) .
$$

ii. Repetition: for all $n \geq 1, w \in \Delta_{n+1}$, and $x \in \mathbb{R}_{+}^{n}$,

$$
\begin{aligned}
& M\left(\left(w_{1}, \ldots, w_{n-1}, w_{n}, w_{n+1}\right),\left(x_{1}, \ldots, x_{n-1}, x_{n}, x_{n}\right)\right) \\
= & M\left(\left(w_{1}, \ldots, w_{n-1}, w_{n}+w_{n+1}\right),\left(x_{1}, \ldots, x_{n-1}, x_{n}\right)\right) .
\end{aligned}
$$

iii. Zero weight: for all $n \geq 1, w \in \Delta_{n}$, and $x \in \mathbb{R}_{+}^{n+1}$,

$$
M\left(\left(w_{1}, \ldots, w_{n}, 0\right),\left(x_{1}, \ldots, x_{n}, x_{n+1}\right)\right)=M\left(\left(w_{1}, \ldots, w_{n}\right),\left(x_{1}, \ldots, x_{n}\right)\right) .
$$

Proof Symmetry is proved by applying functoriality to the bijection $\sigma$. Repetition is proved by applying functoriality to the surjection $\{1, \ldots, n+1\} \rightarrow\{1, \ldots, n\}$ sending $n+1$ to $n$ and fixing all other elements. The zero weight property is proved by applying functoriality to the inclusion $\{1, \ldots, n\} \hookrightarrow\{1, \ldots, n+1\}$.

By functoriality applied to bijections, it makes no difference if we restrict our attention to just one set $\mathbf{n}=\{1, \ldots, n\}$ of each cardinality. Thus, a system of means may be viewed as a sequence of functions $\left(M: \Delta_{n} \times \mathbb{R}_{+}^{n} \rightarrow \mathbb{R}_{+}\right)_{n=1}^{\infty}$ satisfying symmetry, repetition, zero weight, consistency, and monotonicity. To state the multiplicativity axiom we must choose a bijection $\mathbf{m} \times \mathbf{n} \rightarrow \mathbf{m n}$ for each $m$ and $n$, but by symmetry, the axiom is unaffected by that choice.

A third option, in the spirit of [2], construes a system of means as a single function

$$
M: c_{00}^{\Delta} \times c_{00}^{+} \rightarrow \mathbb{R}_{+},
$$

where $c_{00}^{+}$is the set of finitely-supported sequences in $\mathbb{R}_{+}$and $c_{00}^{\Delta}=\left\{w \in c_{00}^{+} \mid \sum w_{i}=1\right\}$. It is to satisfy the evident reformulations of symmetry, repetition, zero weight, consistency, and monotonicity. To state the multiplicativity axiom we must choose a bijection between the set of positive integers and its cartesian square, but again the choice is immaterial.

The next result says that a weighted mean of numbers increases when weight is transferred from a smaller number to a larger one. 
Lemma 2.2 Every system of means $M$ has the transfer property, as follows. Let $n \geq 2$, $w \in \Delta_{n}, x \in \mathbb{R}_{+}^{n}$, and $0 \leq \varepsilon \leq w_{n}$. Suppose that $x_{n} \leq x_{n-1}$. Then

$$
M(w, x) \leq M\left(\left(w_{1}, \ldots, w_{n-2}, w_{n-1}+\varepsilon, w_{n}-\varepsilon\right), x\right) .
$$

Proof We have

$$
\begin{aligned}
M(w, x) & =M\left(\left(w_{1}, \ldots, w_{n-2}, w_{n-1}, \varepsilon, w_{n}-\varepsilon\right),\left(x_{1}, \ldots, x_{n-2}, x_{n-1}, x_{n}, x_{n}\right)\right) \\
& \leq M\left(\left(w_{1}, \ldots, w_{n-2}, w_{n-1}, \varepsilon, w_{n}-\varepsilon\right),\left(x_{1}, \ldots, x_{n-2}, x_{n-1}, x_{n-1}, x_{n}\right)\right) \\
& =M\left(\left(w_{1}, \ldots, w_{n-2}, w_{n-1}+\varepsilon, w_{n}-\varepsilon\right), x\right)
\end{aligned}
$$

by (respectively) repetition, monotonicity, and repetition. (Symmetry is also used, but we will generally let this go unmentioned.)

Lemma 2.3 Every multiplicative system of means $M$ is homogeneous: $M(w, c x)=$ $c M(w, x)$ whenever $n \geq 1, w \in \Delta_{n}, x \in \mathbb{R}_{+}^{n}$ and $c \in \mathbb{R}_{+}$.

Proof We have

$$
M(w, c x)=M((1) \otimes w,(c) \otimes x)=M((1),(c)) \cdot M(w, x)=c M(w, x)
$$

by definition of $\otimes$, multiplicativity, and consistency.

\subsection{Recovering the exponent}

For the rest of Section 2, fix a convex multiplicative system of means $M$. We will prove that $M=M_{p}$ for some $p \in[1, \infty]$.

To find $p$, define $\theta:(0,1) \rightarrow \mathbb{R}_{+}$by

$$
\theta(s)=M((s, 1-s),(1,0))
$$

$(s \in(0,1))$. By multiplicativity and repetition, $\theta\left(s s^{\prime}\right)=\theta(s) \theta\left(s^{\prime}\right)$ for all $s, s^{\prime} \in(0,1)$, and by transfer, $\theta$ is (non-strictly) increasing. If $\theta(s)=0$ for some $s \in(0,1)$ then $\theta(s)=0$ for all $s \in(0,1)$. If not, put $\phi(t)=-\log \theta\left(e^{-t}\right)(t>0)$. Then $\phi$ satisfies the Cauchy functional equation $\phi\left(t+t^{\prime}\right)=\phi(t)+\phi\left(t^{\prime}\right)$, and is increasing, from which it follows easily that $\phi(t)=\alpha t$ for some constant $\alpha \geq 0$ (as in Section 2.1.1 of [1]). Hence $\theta(s)=s^{\alpha}$. But

$$
\begin{aligned}
\theta\left(\frac{1}{2}\right) & =\max \left\{M\left(\left(\frac{1}{2}, \frac{1}{2}\right),(1,0)\right), M\left(\left(\frac{1}{2}, \frac{1}{2}\right),(0,1)\right)\right\} \\
& \geq M\left(\left(\frac{1}{2}, \frac{1}{2}\right),\left(\frac{1}{2}, \frac{1}{2}\right)\right)=M\left((1),\left(\frac{1}{2}\right)\right)=\frac{1}{2}
\end{aligned}
$$

by (respectively) symmetry, convexity, repetition and consistency. So $\theta(s)=s^{\alpha}$ for some $\alpha \in[0,1]$. Put $p=1 / \alpha \in[1, \infty]$ : then $\theta(s)=s^{1 / p}$. (In the case $p=\infty$, we will always understand $1 / p$ to mean 0 .)

\subsection{Applying the $p$-norm characterization theorem}

First we recall Theorem 1.1 of Aubrun and Nechita [2], rephrasing it slightly.

Given an injection $f: I \rightarrow J$ of finite sets, there is an induced map $\mathbb{R}^{I} \rightarrow \mathbb{R}^{J}$, denoted by $x \mapsto f x$. It is defined, for $j \in J$, by $(f x)_{j}=x_{i}$ if $j=f(i)$ for some $i \in I$, and $(f x)_{j}=0$ otherwise. Given $x \in \mathbb{R}^{I}$ and $y \in \mathbb{R}^{J}$, write $x \otimes y=\left(x_{i} y_{j}\right)_{(i, j) \in I \times J} \in \mathbb{R}^{I \times J}$.

A system of norms consists of a norm $\|\cdot\|$ on $\mathbb{R}^{I}$ for each finite set $I$, such that $\|f x\|=$ $\|x\|$ whenever $f: I \rightarrow J$ is an injection of finite sets and $x \in \mathbb{R}^{I}$. It is multiplicative if $\|x \otimes y\|=\|x\|\|y\|$ whenever $x \in \mathbb{R}^{I}$ and $y \in \mathbb{R}^{J}$.

For example, for each $q \in[1, \infty]$ there is a multiplicative system of norms $\|\cdot\|_{q}$ defined by the formula in the Introduction. Theorem 1.1 of [2] states that these are the only ones: 
Theorem 2.4 Every multiplicative system of norms is equal to $\|\cdot\|_{q}$ for some $q \in[1, \infty]$.

We now resume our proof. For a finite set $I$ with $n \geq 1$ elements, denote the uniform distribution on $I$ by $u_{I}=(1 / n)_{i \in I} \in \Delta_{I}$, and define a function $\|\cdot\|: \mathbb{R}^{I} \rightarrow \mathbb{R}_{+}$by

$$
\|x\|=n^{1 / p} M\left(u_{I},\left(\left|x_{i}\right|\right)_{i \in I}\right)
$$

$\left(x \in \mathbb{R}^{I}\right)$. To cover the case $I=\emptyset$, let $\|\cdot\|: \mathbb{R}^{\emptyset} \rightarrow \mathbb{R}_{+}$be the function whose single value is 0 .

When $I=\{1, \ldots, n\}$, we write $u_{I}$ as $u_{n}$. We will use the observation that

$$
n^{-1 / p}=M((1 / n, 1-1 / n),(1,0))=M\left(u_{n},(1,0, \ldots, 0)\right),
$$

by the defining property of $p$ and repetition.

Lemma $2.5\|\cdot\|$ is a norm on $\mathbb{R}^{I}$, for each finite set $I$.

Proof It is enough to prove this when $I=\{1, \ldots, n\}$. For $x \in \mathbb{R}^{n}$ and $c \in \mathbb{R}$, we have $\|c x\|=|c|\|x\|$ by homogeneity. If $x \in \mathbb{R}^{n}$ with $x \neq 0$ then by symmetry we may assume that $x_{1} \neq 0$, and then

$$
\|x\| \geq n^{1 / p} M\left(u_{n},\left(\left|x_{1}\right|, 0, \ldots, 0\right)\right)=\left|x_{1}\right|>0
$$

by monotonicity, homogeneity, and equation (1).

It remains to prove the triangle inequality, or equivalently that the unit ball $B=\{x \in$ $\left.\mathbb{R}^{n} \mid\|x\| \leq 1\right\}$ is convex. Let $x, y \in B$ and $\lambda \in[0,1]$. We must prove that $\lambda x+(1-\lambda) y \in B$. Write $\widetilde{x}=\left(\left|x_{1}\right|, \ldots,\left|x_{n}\right|\right)$, and similarly $\widetilde{y}$; then $\widetilde{x}, \widetilde{y} \in B$, and

$$
\|\lambda x+(1-\lambda) y\| \leq\|\lambda \widetilde{x}+(1-\lambda) \widetilde{y}\|
$$

by monotonicity, so we may assume that $x_{i}, y_{i} \geq 0$ for all $i$.

When $\lambda$ is a dyadic rational, $\lambda x+(1-\lambda) y \in B$ by convexity of $M$. For the general case, let $\varepsilon>0$. Choose a dyadic $\lambda^{\prime} \in[0,1]$ such that $\lambda \leq(1+\varepsilon) \lambda^{\prime}$ and $1-\lambda \leq(1+\varepsilon)\left(1-\lambda^{\prime}\right)$. Then

$$
\|\lambda x+(1-\lambda) y\| \leq\left\|(1+\varepsilon) \lambda^{\prime} x+(1+\varepsilon)\left(1-\lambda^{\prime}\right) y\right\|=(1+\varepsilon)\left\|\lambda^{\prime} x+\left(1-\lambda^{\prime}\right) y\right\| \leq 1+\varepsilon
$$

by monotonicity and homogeneity. But $\varepsilon$ was arbitrary, so $\|\lambda x+(1-\lambda) y\| \leq 1$.

Next I claim that $\|\cdot\|$ is a system of norms. By functoriality, it suffices to show that

$$
\left\|\left(x_{1}, \ldots, x_{n}\right)\right\|=\left\|\left(x_{1}, \ldots, x_{n}, 0\right)\right\|
$$

for all $x \in \mathbb{R}^{n}(n \geq 1)$. By definition of $\|\cdot\|$ and equation (1), this is equivalent to

$$
\begin{aligned}
& M\left(u_{n+1},(1,0, \ldots, 0)\right) \cdot M\left(u_{n},\left(\left|x_{1}\right|, \ldots,\left|x_{n}\right|\right)\right) \\
= & M\left(u_{n},(1,0, \ldots, 0)\right) \cdot M\left(u_{n+1},\left(\left|x_{1}\right|, \ldots,\left|x_{n}\right|, 0\right)\right) .
\end{aligned}
$$

But by multiplicativity, both sides are equal to

$$
M\left(u_{n(n+1)},\left(\left|x_{1}\right|, \ldots,\left|x_{n}\right|, 0, \ldots, 0\right)\right)
$$

proving the claim.

The system of norms $\|\cdot\|$ is, moreover, multiplicative, by multiplicativity of $M$.

Theorem 2.4 now implies that $\|\cdot\|=\|\cdot\|_{q}$ for some $q \in[1, \infty]$. But

$$
\|(1,1)\|=2^{1 / p} M\left(u_{2},(1,1)\right)=2^{1 / p} M((1),(1))=2^{1 / p}
$$

by definition of $\|\cdot\|$, repetition and consistency, whereas $\|(1,1)\|_{q}=2^{1 / q}$, so $p=q$. Hence for all $n \geq 1$ and $x \in \mathbb{R}_{+}^{n}$,

$$
M\left(u_{n}, x\right)=n^{-1 / p}\|x\|_{p}=M_{p}\left(u_{n}, x\right) .
$$




\subsection{The case of uneven weights}

We now know that $M\left(u_{n}, x\right)=M_{p}\left(u_{n}, x\right)$ for all $n \geq 1$ and $x \in \mathbb{R}_{+}^{n}$. Here we deduce the same equation for an arbitrary weighting $w$ in place of $u_{n}$. This will complete the proof.

When the coordinates of $w$ are rational, the equation can be proved by a standard technique, described in Section 2.2 of [4]. Let $n \geq 1, w \in \Delta_{n} \cap \mathbb{Q}^{n}$ and $x \in \mathbb{R}_{+}^{n}$. Write $w_{i}=k_{i} / k$ where $k_{i}$ and $k$ are nonnegative integers; thus, $\sum_{i} k_{i}=k>0$. We have

$$
M(w, x)=M\left(\left(\frac{k_{1}}{k}, \ldots, \frac{k_{n}}{k}\right), x\right)=M\left(u_{k},(\underbrace{x_{1}, \ldots, x_{1}}_{k_{1}}, \ldots, \underbrace{x_{n}, \ldots, x_{n}}_{k_{n}})\right)
$$

by repetition and the zero weight property. (The latter is needed to cover the possibility that $w_{i}=0$ for some values of $i$.) But $M\left(u_{k},-\right)=M_{p}\left(u_{k},-\right)$, so

$$
M(w, x)=\left(\sum_{i=1}^{n} k_{i} \frac{1}{k} x_{i}^{p}\right)^{1 / p}=M_{p}(w, x)
$$

when $p<\infty$, and similarly

$$
M(w, x)=\max _{i: k_{i}>0} x_{i}=\max _{i: w_{i}>0} x_{i}=M_{\infty}(w, x)
$$

when $p=\infty$.

To extend the result to irrational weights, we use an approximation lemma.

Lemma 2.6 Let $w \in \Delta_{n}, x \in \mathbb{R}_{+}^{n}$, and $\delta>0$. Then there exist $w^{\prime}, w^{\prime \prime} \in \Delta_{n} \cap \mathbb{Q}^{n}$ such that

$$
M\left(w^{\prime}, x\right) \geq M(w, x) \geq M\left(w^{\prime \prime}, x\right)
$$

with $\left\|w-w^{\prime}\right\|_{\infty}<\delta$ and $\left\|w-w^{\prime \prime}\right\|_{\infty}<\delta$

Proof We just prove the existence of such a $w^{\prime}$, the argument for $w^{\prime \prime}$ being similar. Suppose without loss of generality that $x_{1} \leq \cdots \leq x_{n}$. Choose $\delta_{1} \in[0, \delta)$ with $w_{1}-\delta_{1} \in \mathbb{Q} \cap \mathbb{R}_{+}$. Then

$$
M\left(\left(w_{1}-\delta_{1}, w_{2}+\delta_{1}, w_{3}, \ldots, w_{n}\right), x\right) \geq M(w, x)
$$

by transfer. Next choose $\delta_{2} \in[0, \delta)$ such that $w_{2}+\delta_{1}-\delta_{2} \in \mathbb{Q} \cap \mathbb{R}_{+}$. Then

$$
M\left(\left(w_{1}-\delta_{1}, w_{2}+\delta_{1}-\delta_{2}, w_{3}+\delta_{2}, w_{4}, \ldots, w_{n}\right), x\right) \geq M\left(\left(w_{1}-\delta_{1}, w_{2}+\delta_{1}, w_{3}, \ldots, w_{n}\right), x\right) .
$$

Continuing in this way, we arrive at

$$
M\left(\left(w_{1}-\delta_{1}, w_{2}+\delta_{1}-\delta_{2}, w_{3}+\delta_{2}-\delta_{3}, \ldots, w_{n-1}+\delta_{n-2}-\delta_{n-1}, w_{n}+\delta_{n-1}\right), x\right) \geq M(w, x) .
$$

Taking $w^{\prime}$ to be the weighting on the left-hand side gives the result.

Now we prove by induction on $n \geq 1$ that $M(w, x)=M_{p}(w, x)$ whenever $w \in \Delta_{n}$ and $x \in \mathbb{R}_{+}^{n}$. It is trivial for $n=1$; suppose that $n \geq 2$. Let $w \in \Delta_{n}$ and $x \in \mathbb{R}_{+}^{n}$. If $w_{i}=0$ for some $i$ then $M(w, x)=M_{p}(w, x)$ by the zero weight property and inductive hypothesis. Suppose, then, that $w_{i}>0$ for all $i$.

Let $\varepsilon>0$. Since $M_{p}(-, x)$ is continuous on $\left\{w^{\prime} \in \Delta_{n} \mid w_{i}^{\prime}>0\right.$ for all $\left.i\right\}$, we may choose $\delta>0$ such that $\left|M_{p}(w, x)-M_{p}\left(w^{\prime}, x\right)\right|<\varepsilon$ for all $w^{\prime}$ such that $\left\|w-w^{\prime}\right\|_{\infty}<\delta$. Choose $w^{\prime}$ as in Lemma 2.6: then

$$
M(w, x) \leq M\left(w^{\prime}, x\right)=M_{p}\left(w^{\prime}, x\right)<M_{p}(w, x)+\varepsilon .
$$

This holds for all $\varepsilon>0$, so $M(w, x) \leq M_{p}(w, x)$. Similarly, applying the other half of Lemma 2.6, $M(w, x) \geq M_{p}(w, x)$. Hence $M(w, x)=M_{p}(w, x)$, completing the proof. 


\section{A variant}

The zero weight property was used only in Section 2.4, and only in order to handle means $M(w, x)$ in which $w_{i}=0$ for some $i$. This suggests a variant of Theorem 1.3 in which all weights are required to be positive and the zero weight property is dropped. This amounts to using $\Delta_{I}^{\circ}=\left\{w \in \Delta_{I} \mid w_{i}>0\right.$ for all $\left.i \in I\right\}$ in place of $\Delta_{I}$, and using only surjections between finite sets.

Thus, a system of positively weighted means is defined just as a system of means was defined, but replacing $\Delta_{I}$ by $\Delta_{I}^{\circ}$ and only demanding functoriality for surjections. For each $p \in[1, \infty]$ there is a convex multiplicative system of positively weighted means, $M_{p}$, defined by restricting the system of means of the same name. By removing all mention of zero weights from the proof above, we obtain:

Theorem 3.1 Every convex multiplicative system of positively weighted means is equal to $M_{p}$ for some $p \in[1, \infty]$.

\section{References}

[1] J. Aczél. Lectures on Functional Equations and their Applications. Academic Press, New York, 1966.

[2] G. Aubrun and I. Nechita. The multiplicative property characterizes $\ell_{p}$ and $L_{p}$ norms. arXiv:1102.2618, 2011.

[3] C. Fernández-González, C. Palazuelos, and D. Pérez-García. The natural rearrangement invariant structure on tensor products. Journal of Mathematical Analysis and Applications, 343:40-47, 2008.

[4] G. Hardy, J. E. Littlewood, and G. Pólya. Inequalities. Cambridge University Press, Cambridge, second edition, 1952. 